\title{
Synthesis and cAMP-dependent phosphodiesterase inhibition of novel thiazoloquinazoline derivatives
}

THEIVENDREN PANNEER SELVAM ${ }^{1 *}$ PALANIRAJAN VIJAYARAJ KUMAR ${ }^{2}$

${ }^{1}$ Department of Biotechnology Acharya Nagarjuna University Guntur-522510, India

2 Department of Pharmacy, College Sadaya, International University Jalan Menara Gading 56000 Cheras Kuala Lumpur, Malaysia
The series of 6,7,8,9-tetrahydro-5H-5-(2'-hydroxyphenyl)-2-(4'-substituted benzylidine)thiazolo $(2,3-b)$ quinazolin$-3(2 \mathrm{H})$-ones $(4 \mathbf{a - j})$ and 6,7,8,9-tetrahydro-5H-5-(2'-hydroxyphenyl)-2-(4'-substituted benzylidine)-3-(4-nitrophenyl amino)thiazoloquinazolines (5a-j) were synthesized by the reported method and evaluated for their phosphodiesterase inhibitory activity. All test compounds exhibited good activity. The structure-activity relationships were also studied. In both series of compounds, electron-withdrawing substitutions showed higher activity. Among the tested compounds, 6,7,8,9-tetrahydro-5H-5-(2'-hydroxyphenyl)-2-(4'-fluorobenzylidine)-3-(4-nitrophenylamino)thiazoloquinazoline (5e), 6,7,8,9-tetrahydro-5H-5-(2'-hydroxyphenyl)-2-(4'-nitrobenzylidine)-3-(4-nitrophenylamino)thiazoloquinazoline (5j) and 6,7,8,9-tetrahydro-5H-5-(2'-hydroxyphenyl)-2-(4'-chlorobenzylidine)-3-(4-nitrophenylamino)thiazoloquinazoline (5f) were found to be more potent than theophylline $\left(I C_{50}\right.$ in $\mathrm{mmol} \mathrm{L}^{-1}$ of $1.34 \pm 0.09$ for $5 \mathbf{f}, 1.44 \pm 0.02$ for $5 \mathbf{e}, 1.52 \pm 0.05$ for $5 \mathbf{j}$ vs. $1.72 \pm 0.09$ for theophylline).

Keywords: thiazoloquinazoline, benzylidinethiazoloquinazoline, nitrophenylaminothiazoloquinazoline, phosphodiesterase inhibitions, SAR

Cyclic 3,5-adenosine monophosphate (cAMP) and cyclic 3,5-guanosine monophosphate (cGMP) are important second messengers that play a central role in mediating a variety of functional responses to hormones and other cellular transmitters (1-4). Sensitivity of the physiological processes regulated by cyclic nucleotides requires precise and rapid regulation of the level of these second messengers according to the requirements of the physiological status of the cell. Precise modulation of the phosphodiesterase (PDE) function is critical for maintaining cyclic nucleotide levels within a narrow concentration range. Due to their key role in the regulation of physiological processes, inhibitors of PDEs can be used as therapeutic tools for various diseases. Relatively less emphasis, however, has been placed on the development of agents that interfere with the

\footnotetext{
* Correspondence; e-mail: tpsphc@gmail.com
} 
catabolism of cAMP and cGMP via inhibition of phosphodiesterase. A variety of nitrogen and sulfur containing heterocycles are reported to inhibit PDE (5-10). Quinazolines and condensed quinazoline such as hoquizil, prazosin and buqineran, possess PDE inhibitory $(11,12)$ antimicrobial $(13)$, antiinflammatory (14), anticonvulsant (15) and antihypertensive (16) activity (Fig. 1). Based on these observations, a hypothetical model has been proposed, taking into account the following broad objectives: to develop new fused heterocycles expected to exert PDE inhibitory activity similar to that of theophylline.<smiles>CCCCN(NC(=O)OCC)N1CCN(c2ncnc3cc(OC)c(OC)cc23)CC1</smiles>

Fig. 1. Quinazoline bearing drugs.

Based on this approach, we attempted to synthesize 6,7,8,9-tetrahydro-5H-5-(2'-hydroxyphenyl)-2-(4'-substituted benzylidine)thiazolo(2,3-b)quinazolin-3(2H)-one (4a-j) and 6,7,8,9-tetrahydro-5H-5-(2'-hydroxyphenyl)-2-(4'-substituted benzylidine)-3-(4-nitrophenylamino)thiazoloquinazolines $(\mathbf{5 a} \mathbf{a} \mathbf{j})$ analogues. The PDE inhibitory activity for all title compounds (4a-j and $\mathbf{5 a - j}$ ) was tested using a standard technique (4).

\section{EXPERIMENTAL}

Bovine heart phosphodiesterase sample was obtained from Sigma (India) and theophylline, as a reference compound, from German Remedies (India).

Melting points were taken in an open capillary tube and were uncorrected. IR spectra were recorded in $\mathrm{KBr}$ pellets (ABB Bomem FT-IR spectrometer MB 104, ABB Limited, India). The ${ }^{1} \mathrm{H}-\mathrm{NMR}$ spectra were recorded in $\mathrm{CDCl}_{3}$ (Bruker $400 \mathrm{NMR}$ spectrometer, IET Limited) with TMS as internal reference. Mass spectral data were recorded with a quadrupol mass spectrometer (Shimadzu GC MS QP 5000, Japan). Microanalyses were performed using a Vario EL V300 elemental analyzer (Elemental Analysensysteme GmbH, Germany). The purity of the compounds was checked by TLC on pre-coated $\mathrm{SiO}_{2}$ gel $\left(\mathrm{HF}_{254}\right)$ aluminium plates (Merck, USA) using ethyl acetate/benzene (1:3) and visualized in a UV chamber. Physico-chemical and spectral data results of these compounds are shown in Table I. 
T. P. Selvam and P. V. Kumar: Synthesis and cAMP-dependent phosphodiesterase inhibition of novel thiazoloquinazoline derivatives, Acta Pharm. 61 (2011) 103-113.

Table I. Spectral data of synthesized compounds

\begin{tabular}{|c|c|c|c|c|c|c|c|c|}
\hline \multirow[t]{2}{*}{ Compd. } & \multirow[t]{2}{*}{$\begin{array}{c}\text { Yield } \\
(\%)\end{array}$} & \multirow[t]{2}{*}{$\begin{array}{l}\text { M. p. } \\
\left({ }^{\circ} \mathrm{C}\right)\end{array}$} & \multirow[t]{2}{*}{$\begin{array}{l}\text { IR }(\mathrm{KBr}) \\
\left(v, \mathrm{~cm}^{-1}\right)\end{array}$} & \multirow[t]{2}{*}{${ }^{1} \mathrm{H} \operatorname{NMR}(\delta, \mathrm{ppm})$} & \multirow[t]{2}{*}{$m / z$} & \multicolumn{3}{|c|}{$\begin{array}{l}\text { Molecular formula } \\
\text { Analysis: } \\
\text { calcd./found (\%) }\end{array}$} \\
\hline & & & & & & $\mathrm{C}$ & $\mathrm{H}$ & $\mathrm{N}$ \\
\hline & & & $3402(\mathrm{O}-\mathrm{H})$ & 6.61-6.89 (m, 4H Ar-H), 5.71 & & \multicolumn{3}{|c|}{$\mathrm{C}_{16} \mathrm{H}_{16} \mathrm{~N}_{2} \mathrm{O}_{2} \mathrm{~S}$} \\
\hline 3 & 71 & 153-155 & $\begin{array}{l}3046(\mathrm{Ar}-\mathrm{CH}), \\
1719(\mathrm{C}=\mathrm{O}), \\
1462(\mathrm{C}=\mathrm{C})\end{array}$ & $\begin{array}{l}(\mathrm{s}, 1 \mathrm{H},-\mathrm{CH}) 9.91(\mathrm{~s}, 1 \mathrm{H}, \\
\text { Ar-OH), } 3.76\left(\mathrm{~s}, 2 \mathrm{H},-\mathrm{CH}_{2}\right) \\
1.6-2.42\left(\mathrm{~m}, 8 \mathrm{H}, 4 \times \mathrm{CH}_{2}\right)\end{array}$ & $\begin{array}{l}{[\mathrm{M}+]} \\
(06)\end{array}$ & $\begin{array}{l}63.98 \\
63.92\end{array}$ & $\begin{array}{l}5.37 \\
5.28\end{array}$ & $\begin{array}{l}9.32 \\
9.30\end{array}$ \\
\hline $4 a$ & 82 & 159-161 & $\begin{array}{l}3474(\mathrm{O}-\mathrm{H}), \\
3093(\mathrm{Ar}-\mathrm{CH}), \\
1725(\mathrm{C}=\mathrm{O}), \\
1461(\mathrm{C}=\mathrm{C})\end{array}$ & $\begin{array}{l}\text { 6.92-7.56 (m, 9H, Ar-H), } 6.63 \\
(\mathrm{~s}, 1 \mathrm{H},=\mathrm{CH}), 5.81(\mathrm{~s}, 1 \mathrm{H} \\
\mathrm{H}-5), 9.74(\mathrm{~s}, 1 \mathrm{H}, \mathrm{Ar}-\mathrm{OH}) \\
1.58-2.67\left(\mathrm{~m}, 8 \mathrm{H}, 4 \times \mathrm{CH}_{2}\right)\end{array}$ & $\begin{array}{l}377 \\
{[\mathrm{M}+]} \\
(38)\end{array}$ & $\begin{array}{r}\mathrm{C}_{23} \\
71.11 \\
71.19\end{array}$ & $\begin{array}{l}\mathrm{H}_{20} \mathrm{~N}_{2} \mathrm{C} \\
5.19 \\
5.26\end{array}$ & $\begin{array}{l}{ }_{2} S \\
7.21 \\
7.14\end{array}$ \\
\hline $4 b$ & 79 & $176-178$ & $\begin{array}{l}3442(\mathrm{O}-\mathrm{H}), \\
3041(\mathrm{Ar}-\mathrm{CH}), \\
1723(\mathrm{C}=\mathrm{O}), \\
1431(\mathrm{C}=\mathrm{C})\end{array}$ & $\begin{array}{l}\text { 6.81-7.71 (m, 8H, Ar-H), } 6.73 \\
(\mathrm{~s}, 1 \mathrm{H},=\mathrm{CH}), 5.71(\mathrm{~s}, 1 \mathrm{H} \\
\mathrm{H}-5), 9.76(\mathrm{~s}, 2 \mathrm{H}, \mathrm{Ar}-\mathrm{OH}) \\
1.61-2.35\left(\mathrm{~m}, 8 \mathrm{H}, 4 \times \mathrm{CH}_{2}\right)\end{array}$ & $\begin{array}{l}404 \\
{[\mathrm{M}+]} \\
(49)\end{array}$ & $\begin{array}{r}\mathrm{C}_{23} \\
68.30 \\
68.37\end{array}$ & $\begin{array}{l}\mathrm{H}_{20} \mathrm{~N}_{2} \mathrm{C} \\
4.98 \\
4.87\end{array}$ & $\begin{array}{l}{ }_{3} S \\
6.93 \\
6.99\end{array}$ \\
\hline $4 c$ & 78 & 183-185 & $\begin{array}{l}3476(\mathrm{O}-\mathrm{H}), \\
3096(\mathrm{Ar}-\mathrm{CH}), \\
1728(\mathrm{C}=\mathrm{O}), \\
1468(\mathrm{C}=\mathrm{C})\end{array}$ & $\begin{array}{l}6.96-7.54(\mathrm{~m}, 8 \mathrm{H}, \mathrm{Ar}-\mathrm{H}), 6.67 \\
(\mathrm{~s}, 1 \mathrm{H},=\mathrm{CH}), 5.83(\mathrm{~s}, 1 \mathrm{H}, \\
\mathrm{H}-5), 9.84(\mathrm{~s}, 1 \mathrm{H}, \mathrm{Ar}-\mathrm{OH}) \\
3.75\left(\mathrm{~s}, 3 \mathrm{H}-\mathrm{OCH}_{3}\right), 1.58-2.62 \\
\left(\mathrm{~m}, 8 \mathrm{H}, 4 \times \mathrm{CH}_{2}\right)\end{array}$ & $\begin{array}{l}418 \\
{[\mathrm{M}+]} \\
(67)\end{array}$ & $\begin{array}{r}\mathrm{C}_{24} \\
68.88 \\
68.90\end{array}$ & $\begin{array}{l}\mathrm{H}_{22} \mathrm{~N}_{2} \mathrm{C} \\
5.30 \\
5.33\end{array}$ & $\begin{array}{l}{ }_{3} S \\
6.69 \\
6.72\end{array}$ \\
\hline $4 d$ & 76 & 186-188 & $\begin{array}{l}3448(\mathrm{O}-\mathrm{H}), \\
3049(\mathrm{Ar}-\mathrm{CH}), \\
1721(\mathrm{C}=\mathrm{O}), \\
1434(\mathrm{C}=\mathrm{C})\end{array}$ & $\begin{array}{l}6.86-7.74(\mathrm{~m}, 8 \mathrm{H}, \mathrm{Ar}-\mathrm{H}), 6.72 \\
(\mathrm{~s}, 1 \mathrm{H},=\mathrm{CH}), 5.76(\mathrm{~s}, 1 \mathrm{H}, \\
\mathrm{H}-5), 9.76(\mathrm{~s}, 1 \mathrm{H}, \mathrm{Ar}-\mathrm{OH}) \\
2.20\left(\mathrm{~s}, 3 \mathrm{H}-\mathrm{CH}_{3}\right), 1.62-2.32 \\
\left(\mathrm{~m}, 8 \mathrm{H}, 4 \times \mathrm{CH}_{2}\right)\end{array}$ & $\begin{array}{l}402 \\
{[\mathrm{M}+]} \\
(62)\end{array}$ & $\begin{array}{r}\mathrm{C}_{24} \\
71.00 \\
69.87\end{array}$ & $\begin{array}{l}\mathrm{H}_{22} \mathrm{~N}_{2} \mathrm{C} \\
5.51 \\
5.32\end{array}$ & $\begin{array}{l}{ }_{2} S \\
6.96 \\
6.74\end{array}$ \\
\hline $4 e$ & 69 & 153-155 & $\begin{array}{l}3437(\mathrm{O}-\mathrm{H}), \\
3026(\mathrm{Ar}-\mathrm{CH}), \\
1729(\mathrm{C}=\mathrm{O}), \\
1522(\mathrm{C}=\mathrm{C})\end{array}$ & $\begin{array}{l}\text { 6.63-7.32 (m, 8H, Ar-H), } 6.38 \\
(\mathrm{~s}, 1 \mathrm{H},=\mathrm{CH}), 5.87(\mathrm{~s}, 1 \mathrm{H} \\
\mathrm{H}-5), 9.94(\mathrm{~s}, 1 \mathrm{H}, \mathrm{Ar}-\mathrm{OH}) \\
1.34-2.33\left(\mathrm{~m}, 8 \mathrm{H}, 4 \times \mathrm{CH}_{2}\right)\end{array}$ & $\begin{array}{l}406 \\
{[\mathrm{M}+]} \\
(52)\end{array}$ & $\begin{array}{r}\mathrm{C}_{23} \mathrm{H} \\
67.96 \\
67.97\end{array}$ & $\begin{array}{c}\mathrm{H}_{19} \mathrm{FN}_{2} \\
4.71 \\
4.73\end{array}$ & $\begin{array}{l}\mathrm{O}_{2} \mathrm{~S} \\
6.89 \\
6.87\end{array}$ \\
\hline $4 f$ & 65 & 157-159 & $\begin{array}{l}3431(\mathrm{O}-\mathrm{H}), \\
3021(\mathrm{Ar}-\mathrm{CH}), \\
1722(\mathrm{C}=\mathrm{O}), \\
1527(\mathrm{C}=\mathrm{C}), \\
816(\mathrm{C}-\mathrm{Cl})\end{array}$ & $\begin{array}{l}\text { 6.61-7.32 (m, 8H, Ar-H), } 6.32 \\
(\mathrm{~s}, 1 \mathrm{H},=\mathrm{CH}), 5.89(\mathrm{~s}, 1 \mathrm{H} \\
\mathrm{H}-5), 9.97(\mathrm{~s}, 1 \mathrm{H}, \mathrm{Ar}-\mathrm{OH}) \\
1.32-2.37\left(\mathrm{~m}, 8 \mathrm{H}, 4 \times \mathrm{CH}_{2}\right)\end{array}$ & $\begin{array}{l}424 \\
{[\mathrm{M}+2]} \\
(73)\end{array}$ & $\begin{array}{c}\mathrm{C}_{23} \mathrm{H} \\
65.32 \\
65.44\end{array}$ & $\begin{array}{c}{ }_{19} \mathrm{ClN}, \\
4.53 \\
4.41\end{array}$ & $\begin{array}{l}\mathrm{O}_{2} \mathrm{~S} \\
6.62 \\
6.67\end{array}$ \\
\hline $4 g$ & 75 & 184-186 & $\begin{array}{l}3447(\mathrm{O}-\mathrm{H}), \\
3025(\mathrm{Ar}-\mathrm{CH}), \\
1716(\mathrm{C}=\mathrm{O}), \\
1523(\mathrm{C}=\mathrm{C}), \\
823(\mathrm{C}-\mathrm{Br})\end{array}$ & $\begin{array}{l}6.73-7.29(\mathrm{~m}, 8 \mathrm{H}, \mathrm{Ar}-\mathrm{H}), 6.48 \\
(\mathrm{~s}, 1 \mathrm{H},=\mathrm{CH}), 5.73(\mathrm{~s}, 1 \mathrm{H} \\
\mathrm{H}-5), 9.89(\mathrm{~s}, 1 \mathrm{H}, \mathrm{Ar}-\mathrm{OH}) \\
1.26-2.32\left(\mathrm{~m}, 8 \mathrm{H}, 4 \times \mathrm{CH}_{2}\right)\end{array}$ & $\begin{array}{l}468 \\
{[\mathrm{M}+2]} \\
(91)\end{array}$ & $\begin{array}{c}\mathrm{C}_{23} \mathrm{H} \\
59.11 \\
59.14\end{array}$ & $\begin{array}{c}{ }_{19} \mathrm{BrN}_{2} \\
4.10 \\
4.13\end{array}$ & $\begin{array}{l}\mathrm{O}_{2} \mathrm{~S} \\
5.99 \\
5.97\end{array}$ \\
\hline $4 h$ & 81 & 185-187 & $\begin{array}{l}3452(\mathrm{O}-\mathrm{H}), \\
3059(\mathrm{Ar}-\mathrm{CH}), \\
1727(\mathrm{C}=\mathrm{O}), \\
1439(\mathrm{C}=\mathrm{C})\end{array}$ & $\begin{array}{l}6.82-7.75(\mathrm{~m}, 6 \mathrm{H}, \mathrm{Ar}-\mathrm{H}), 6.71 \\
(\mathrm{~s}, 1 \mathrm{H},=\mathrm{CH}), 5.73(\mathrm{~s}, 1 \mathrm{H}, \\
\mathrm{H}-5), 9.84(\mathrm{~s}, 1 \mathrm{H}, \mathrm{Ar}-\mathrm{OH}) \\
2.23\left(\mathrm{~s}, 9 \mathrm{H}-\mathrm{OCH}_{3}\right), 1.64-2.38 \\
\left(\mathrm{~m}, 8 \mathrm{H}, 4 \times \mathrm{CH}_{2}\right)\end{array}$ & $\begin{array}{l}478 \\
{[\mathrm{M}+]} \\
(100)\end{array}$ & $\begin{array}{r}C_{26} \\
65.25 \\
65.29\end{array}$ & $\begin{array}{c}\mathrm{H}_{26} \mathrm{~N}_{2} \mathrm{C} \\
5.48 \\
5.32\end{array}$ & $\begin{array}{l}{ }_{5} S \\
5.85 \\
5.89\end{array}$ \\
\hline
\end{tabular}




\begin{tabular}{|c|c|c|c|c|c|c|c|c|}
\hline \multirow[b]{2}{*}{$4 \mathrm{i}$} & \multirow[b]{2}{*}{79} & \multirow[b]{2}{*}{ 181-183 } & \multirow[b]{2}{*}{$\begin{array}{l}3450(\mathrm{O}-\mathrm{H}), \\
3051(\mathrm{Ar}-\mathrm{CH}), \\
1724(\mathrm{C}=\mathrm{O}) \\
1437(\mathrm{C}=\mathrm{C})\end{array}$} & \multirow{2}{*}{$\begin{array}{l}\text { 6.89-7.76 (m, 7H, Ar-H), } \\
6.74(\mathrm{~s}, 1 \mathrm{H},=\mathrm{CH}), 5.78 \\
(\mathrm{~s}, 1 \mathrm{H}, \mathrm{H}-5), 9.84(\mathrm{~s}, 1 \mathrm{H} \\
\mathrm{Ar}-\mathrm{OH}), 2.23\left(\mathrm{~s}, 6 \mathrm{H}-\mathrm{CH}_{3}\right) \\
1.62-2.32\left(\mathrm{~m}, 8 \mathrm{H}, 4 \times \mathrm{CH}_{2}\right)\end{array}$} & \multirow[b]{2}{*}{$\begin{array}{l}416 \\
{[\mathrm{M}+]} \\
(09)\end{array}$} & \multicolumn{3}{|c|}{$\mathrm{C}_{25} \mathrm{H}_{24} \mathrm{~N}_{2} \mathrm{O}_{2} \mathrm{~S}$} \\
\hline & & & & & & $\begin{array}{l}72.09 \\
72.12\end{array}$ & $\begin{array}{l}5.81 \\
5.79\end{array}$ & $\begin{array}{l}6.73 \\
6.75\end{array}$ \\
\hline $4 \mathbf{j}$ & 79 & $184-186$ & $\begin{array}{l}3441(\mathrm{O}-\mathrm{H}), \\
3042(\mathrm{Ar}-\mathrm{CH}) \\
1723(\mathrm{C}=\mathrm{O}) \\
1435(\mathrm{C}=\mathrm{C})\end{array}$ & $\begin{array}{l}6.82-7.71(\mathrm{~m}, 8 \mathrm{H}, \mathrm{Ar}-\mathrm{H}), 6.77 \\
(\mathrm{~s}, 1 \mathrm{H},=\mathrm{CH}), 5.72(\mathrm{~s}, 1 \mathrm{H}, \\
\mathrm{H}-5), 9.71(\mathrm{~s}, 1 \mathrm{H}, \mathrm{Ar}-\mathrm{OH}) \\
1.64-2.36\left(\mathrm{~m}, 8 \mathrm{H}, 4 \times \mathrm{CH}_{2}\right)\end{array}$ & $\begin{array}{l}433 \\
{[\mathrm{M}+]} \\
(74)\end{array}$ & $\begin{array}{r}\mathrm{C}_{23} \\
63.73 \\
63.81\end{array}$ & $\begin{array}{l}\mathrm{H}_{19} \mathrm{~N}_{3} \mathrm{C} \\
4.42 \\
4.54\end{array}$ & $\begin{array}{l}\mathrm{O}_{4} \mathrm{~S} \\
9.69 \\
9.52\end{array}$ \\
\hline $5 a$ & 78 & 157-159 & $\begin{array}{l}3461(\mathrm{O}-\mathrm{H}), \\
3029(\mathrm{Ar}-\mathrm{CH}), \\
1492(\mathrm{C}=\mathrm{C}) \\
1316(\mathrm{~N}-\mathrm{H} \\
\text { bending), } \\
3391(\mathrm{~N}-\mathrm{H} \\
\text { stretching) }\end{array}$ & $\begin{array}{l}\text { 6.74-7.13 }(\mathrm{m}, 13 \mathrm{H}, \mathrm{Ar}-\mathrm{H}) \\
6.32(\mathrm{~s}, 1 \mathrm{H},=\mathrm{CH}), 5.59(\mathrm{~s}, \\
1 \mathrm{H}, \mathrm{H}-5), 9.81(\mathrm{~s}, 1 \mathrm{H}, \mathrm{Ar}-\mathrm{OH}) \\
4.42(\mathrm{~s}, 1 \mathrm{H}, \text { thiazole }), 7.26 \\
(\mathrm{~s}, 1 \mathrm{H}, \mathrm{N}-\mathrm{H}), 1.46-2.42 \\
\left(\mathrm{~m}, 8 \mathrm{H}, 4 \times \mathrm{CH}_{2}\right)\end{array}$ & $\begin{array}{l}510 \\
{[\mathrm{M}+]} \\
(36)\end{array}$ & $\begin{array}{r}C_{29} \\
68.21 \\
68.26\end{array}$ & $\begin{array}{l}\mathrm{H}_{26} \mathrm{~N}_{4} \mathrm{C} \\
5.13 \\
5.19\end{array}$ & $\begin{array}{l}\mathrm{O}_{3} \mathrm{~S} \\
10.97 \\
10.82\end{array}$ \\
\hline $5 b$ & 72 & $151-153$ & $\begin{array}{l}3467(\mathrm{O}-\mathrm{H}), \\
3021(\mathrm{Ar}-\mathrm{CH}), \\
1497(\mathrm{C}=\mathrm{C}) \\
1312(\mathrm{~N}-\mathrm{H} \\
\text { bending), } \\
3391(\mathrm{~N}-\mathrm{H} \\
\text { stretching) }\end{array}$ & $\begin{array}{l}6.74-7.29(\mathrm{~m}, 12 \mathrm{H}, \mathrm{Ar}-\mathrm{H}) \\
6.36(\mathrm{~s}, 1 \mathrm{H},=\mathrm{CH}), 5.62(\mathrm{~s}, \\
1 \mathrm{H}, \mathrm{H}-5), 9.87(\mathrm{~s}, 2 \mathrm{H}, \mathrm{Ar}-\mathrm{OH}) \\
4.46(\mathrm{~s}, 1 \mathrm{H}, \text { thiazole }), 7.23 \\
(\mathrm{~s}, 1 \mathrm{H}, \mathrm{N}-\mathrm{H}), 1.46-2.42 \\
\left(\mathrm{~m}, 8 \mathrm{H}, 4 \times \mathrm{CH}_{2}\right)\end{array}$ & $\begin{array}{l}526 \\
{[\mathrm{M}+]} \\
(21)\end{array}$ & $\begin{array}{r}\mathrm{C}_{29} \\
66.14 \\
66.22\end{array}$ & $\begin{array}{l}\mathrm{H}_{26} \mathrm{~N}_{4} \mathrm{C} \\
4.98 \\
4.88\end{array}$ & $\begin{array}{l}\mathrm{O}_{4} \mathrm{~S} \\
10.64 \\
10.69\end{array}$ \\
\hline $5 c$ & 76 & $156-158$ & $\begin{array}{l}3464(\mathrm{O}-\mathrm{H}), \\
3027(\mathrm{Ar}-\mathrm{CH}), \\
1494(\mathrm{C}=\mathrm{C}) \\
1306(\mathrm{~N}-\mathrm{H} \\
\text { bending), } \\
3396(\mathrm{~N}-\mathrm{H} \\
\text { stretching) }\end{array}$ & $\begin{array}{l}6.72-7.23(\mathrm{~m}, 12 \mathrm{H}, \mathrm{Ar}-\mathrm{H}) \\
6.36(\mathrm{~s}, 1 \mathrm{H},=\mathrm{CH}), 5.62(\mathrm{~s}, \\
1 \mathrm{H}, \mathrm{H}-5), 9.87(\mathrm{~s}, 1 \mathrm{H}, \\
\text { Ar-OH), } 4.46(\mathrm{~s}, 1 \mathrm{H}, \\
\text { thiazole }), 3.78\left(\mathrm{~s}, 3 \mathrm{H}-\mathrm{OCH}_{3}\right) \\
7.29(\mathrm{~s}, 1 \mathrm{H}, \mathrm{N}-\mathrm{H}), 1.46-2.42 \\
\left(\mathrm{~m}, 8 \mathrm{H}, 4 \times \mathrm{CH}_{2}\right)\end{array}$ & $\begin{array}{l}540 \\
{[\mathrm{M}+]} \\
(76)\end{array}$ & $\begin{array}{r}C_{30} \\
66.65 \\
66.67\end{array}$ & $\begin{array}{l}\mathrm{H}_{28} \mathrm{~N}_{4} \mathrm{C} \\
5.22 \\
5.25\end{array}$ & $\begin{array}{l}\mathrm{O}_{4} \mathrm{~S} \\
10.36 \\
10.38\end{array}$ \\
\hline $5 d$ & 76 & 192-193 & $\begin{array}{l}3438(\mathrm{O}-\mathrm{H}), \\
3024(\mathrm{Ar}-\mathrm{CH}), \\
1412(\mathrm{C}=\mathrm{C}) \\
1322(\mathrm{~N}-\mathrm{H} \\
\text { bending }), \\
3310(\mathrm{~N}-\mathrm{H} \\
\text { stretching })\end{array}$ & $\begin{array}{l}\text { 6.69-7.24 }(\mathrm{m}, 12 \mathrm{H}, \mathrm{Ar}-\mathrm{H}) \\
6.28(\mathrm{~s}, 1 \mathrm{H},=\mathrm{CH}), 5.72 \\
(\mathrm{~s}, 1 \mathrm{H}, \mathrm{H}-5), 9.82(\mathrm{~s}, 1 \mathrm{H}, \\
\text { Ar-OH), } 4.45(\mathrm{~s}, 1 \mathrm{H}, \\
\text { thiazole), } 2.28\left(\mathrm{~s}, 3 \mathrm{H},-\mathrm{CH}_{3}\right) \\
7.69(\mathrm{~s}, 1 \mathrm{H}, \mathrm{N}-\mathrm{H}), 1.36-2.41 \\
\left(\mathrm{~m}, 8 \mathrm{H}, 4 \times \mathrm{CH}_{2}\right)\end{array}$ & $\begin{array}{l}524 \\
{[\mathrm{M}+]} \\
(82)\end{array}$ & $\begin{array}{r}C_{30} \\
68.68 \\
68.65\end{array}$ & $\begin{array}{l}\mathrm{H}_{28} \mathrm{~N}_{4} \mathrm{C} \\
5.38 \\
5.36\end{array}$ & $\begin{array}{l}\mathrm{O}_{3} \mathrm{~S} \\
10.68 \\
10.70\end{array}$ \\
\hline $5 e$ & 89 & $184-186$ & $\begin{array}{l}3449(\mathrm{O}-\mathrm{H}), \\
3026(\mathrm{Ar}-\mathrm{CH}), \\
1524(\mathrm{C}=\mathrm{C}), \\
1316(\mathrm{~N}-\mathrm{H} \\
\text { bending), } \\
3319(\mathrm{~N}-\mathrm{H} \\
\text { stretching), } \\
821(\mathrm{C}-\mathrm{F})\end{array}$ & $\begin{array}{l}6.74-7.32(\mathrm{~m}, 12 \mathrm{H}, \mathrm{Ar}-\mathrm{H}) \\
6.23(\mathrm{~s}, 1 \mathrm{H},=\mathrm{CH}), 5.84(\mathrm{~s}, \\
1 \mathrm{H}, \mathrm{H}-5), 9.96(\mathrm{~s}, 1 \mathrm{H}, \mathrm{Ar}-\mathrm{OH}) \\
4.42(\mathrm{~s}, 1 \mathrm{H}, \text { thiazole }), 7.34 \\
(\mathrm{~s}, 1 \mathrm{H}, \mathrm{N}-\mathrm{H}), 1.24-2.32(\mathrm{~m}, \\
\left.8 \mathrm{H}, 4 \times \mathrm{CH}_{2}\right)\end{array}$ & $\begin{array}{l}528 \\
{[\mathrm{M}+]} \\
(100)\end{array}$ & $\begin{array}{r}\mathrm{C}_{29} \mathrm{H} \\
65.89 \\
65.91\end{array}$ & $\begin{array}{l}\mathrm{H}_{25} \mathrm{FN}_{4} \\
4.77 \\
4.79\end{array}$ & $\begin{array}{l}{ }_{4} \mathrm{O}_{3} \mathrm{~S} \\
10.60 \\
10.62\end{array}$ \\
\hline $5 f$ & 72 & $164-166$ & $\begin{array}{l}3445(\mathrm{O}-\mathrm{H}), \\
3025(\mathrm{Ar}-\mathrm{CH}), \\
1523(\mathrm{C}=\mathrm{C}), \\
1315(\mathrm{~N}-\mathrm{H} \\
\text { bending), } \\
3320(\mathrm{~N}-\mathrm{H} \\
\text { stretching), } \\
829(\mathrm{C}-\mathrm{Cl})\end{array}$ & $\begin{array}{l}6.71-7.35(\mathrm{~m}, 12 \mathrm{H}, \mathrm{Ar}-\mathrm{H}) \\
6.23(\mathrm{~s}, 1 \mathrm{H},=\mathrm{CH}), 5.84(\mathrm{~s}, \\
1 \mathrm{H}, \mathrm{H}-5), 9.96(\mathrm{~s}, 1 \mathrm{H}, \\
\text { Ar-OH), } 4.42(\mathrm{~s}, 1 \mathrm{H}, \\
\text { thiazole }), 7.16(\mathrm{~s}, 1 \mathrm{H}, \mathrm{N}-\mathrm{H}) \\
1.24-2.32\left(\mathrm{~m}, 8 \mathrm{H}, 4 \times \mathrm{CH}_{2}\right)\end{array}$ & $\begin{array}{l}547 \\
{[\mathrm{M}+2]} \\
(41)\end{array}$ & $\begin{array}{r}\mathrm{C}_{29} \mathrm{H} \\
63.90 \\
63.84\end{array}$ & $\begin{array}{c}{ }_{25} \mathrm{ClN} \\
4.62 \\
4.67\end{array}$ & $\begin{array}{l}{ }_{4} \mathrm{O}_{3} \mathrm{~S} \\
10.28 \\
10.30\end{array}$ \\
\hline
\end{tabular}


T. P. Selvam and P. V. Kumar: Synthesis and cAMP-dependent phosphodiesterase inhibition of novel thiazoloquinazoline derivatives, Acta Pharm. 61 (2011) 103-113.

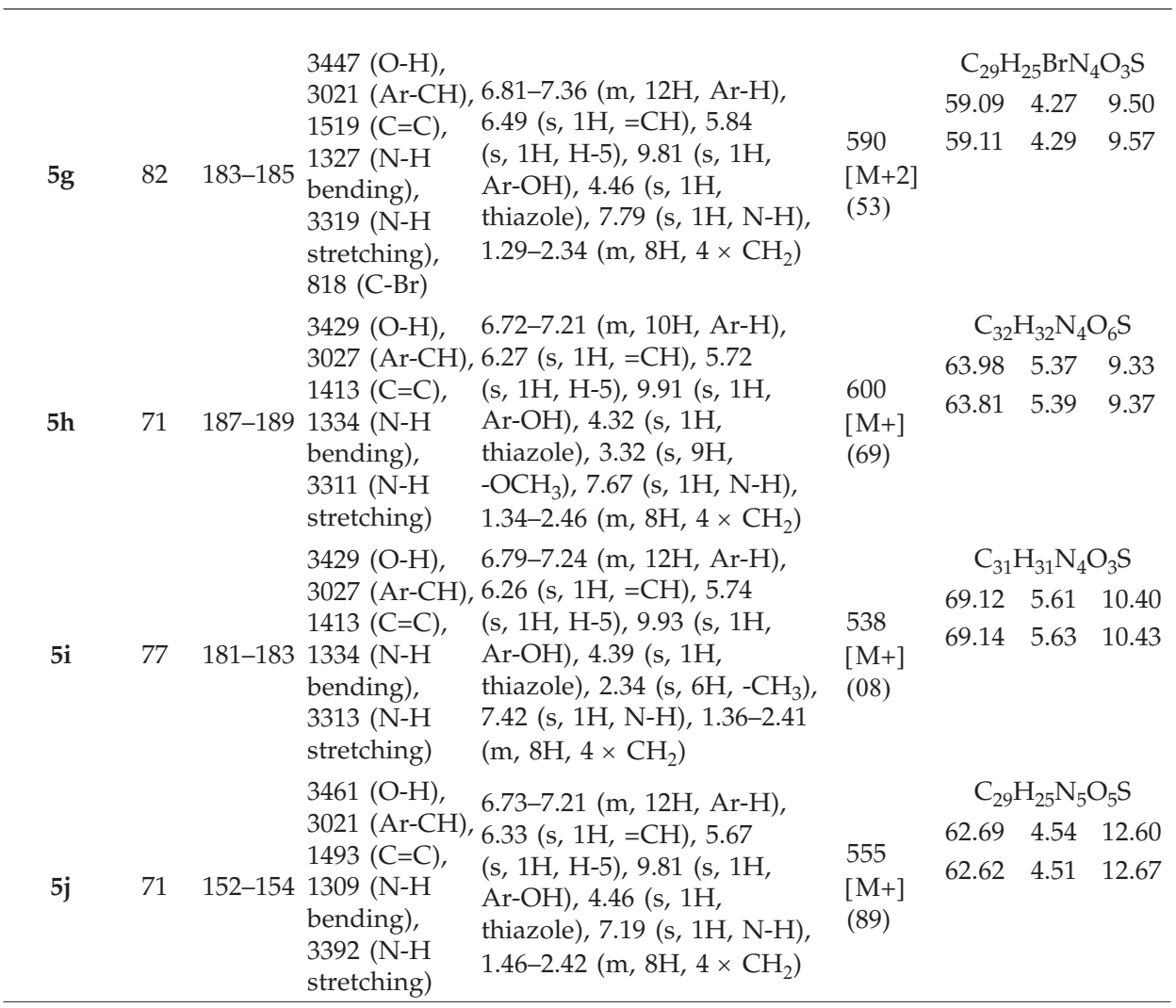

\section{Syntheses}

The target novel thiazoloquinazoline derivatives were synthesized by the previously reported method (17). The key intermediate and target compounds are illustrated in Scheme 1.

2-Hydroxybenzylidine cyclohexanone (1). - Equimolar quantities of each cyclohexanone $(0.039 \mathrm{~mol})$ and salicylaldehyde $(0.039 \mathrm{~mol})$ were taken in a beaker; to this, sodium hydroxide solution was added to make the solution alkaline. This was shaken and kept aside. The solid thus obtained was filtered, washed with water and recrystallized from absolute ethanol.

4-Hydroxy phenyl 3,4,5,6,7,8-hexahydroquinazolin-2-thione (2). - A mixture of 2-hydroxybenzylidine cyclohexanone $(\mathbf{1})(0.039 \mathrm{~mol})$, thiourea $(0.03 \mathrm{~mol})$ and potassium hydroxide $(2.5 \mathrm{~g})$ in $95 \%$ ethanol $(100 \mathrm{~mL})$ was heated under reflux for $3 \mathrm{~h}$. The reaction mixture was concentrated to half its volume, diluted with water, then acidified with glacial acetic acid and kept overnight. The solid thus obtained was filtered, washed with water and recrystallized from ethanol to give 2 . 
T. P. Selvam and P. V. Kumar: Synthesis and cAMP-dependent phosphodiesterase inhibition of novel thiazoloquinazoline derivatives, Acta Pharm. 61 (2011) 103-113.

6,7,8,9-Tetrahydro-5H-5-(2'-hydroxyphenyl)thiazolo(2,3-b)quinazolin-3(2H)-one (3). - Chloroacetic acid $(0.096 \mathrm{~mol})$ was melted on a water bath and $2(0.009 \mathrm{~mol})$ was added portionwise to maintain homogeneity. The homogeneous mixture was heated on a water bath for $30 \mathrm{~min}$ and kept overnight. The solid thus obtained was washed with water and recrystallized from ethanol to give 3 .

6,7,8,9-Tetrahydro-5H-5-(2'-hydroxyphenyl)-2-(4'-substituted benzylidine)thiazolo(2,3-b) quinazolin-3(2H)-ones $(4 a-j)$. - A mixture of 3 (0.002 mol), substituted benzaldehyde $(0.002$ $\mathrm{mol})$ and anhydrous sodium acetate $(0.002 \mathrm{~mol})$ in glacial acetic acid $(10 \mathrm{~mL})$ was heated under reflux for $4 \mathrm{~h}$. The reaction mixture was kept overnight and the solid thus separated was filtered, washed with water and recrystallized from ethanol to furnish 6,7,8,9-tetrahydro-5H-5-(2'-hydroxyphenyl)-2-(4'-substituted benzylidine) thiazolo $(2,3-b)$ quinazolin-3(2H)-ones $(\mathbf{4 a - j})$.

6,7,8,9-Tetrahydro-5H-5-(2'-hydroxyphenyl)-2-(4'-substituted benzylidine)-3-(4-nitrophenylamino)thiazoloquinazolines $(5 a-j)$. - A reaction mixture of the respective quinazolin- $3(2 \mathrm{H})$-one $4 \mathbf{a}-\mathbf{j}(0.004 \mathrm{~mol})$ was dissolved in $(0.004 \mathrm{~mol})$ thionyl chloride. To this, DMF $(0.004 \mathrm{~mol})$ was added to get chloro derivates. The reaction mixture was then coupled with $p$-nitroanilines $(0.004 \mathrm{~mol})$ in DMF at $80^{\circ} \mathrm{C}$ for $3 \mathrm{~h}$. After the mixture was cooled, the precipitate was filtered, recrystallized from ethanol, and dried to give the target compounds 6,7,8,9-tetrahydro-5H-5-(2'-hydroxyphenyl)-2-(4'-substituted

benzylidine)-3-(4-nitrophenylamino)thiazoloquinazolines (5a-j).

\section{PDE inhibition assay} (2).

Inhibition of bovine heart phosphodiesterase was determined by the known method

PDE activity was calculated by measuring the production of inorganic phosphates in the presence of an excess of 5-nucleotidase. Each sample contained $1 \mathrm{mmol} \mathrm{L}^{-1} \mathrm{cAMP}_{\text {, }}$ $3 \mathrm{mmol} \mathrm{L}^{-1} \mathrm{MgSO}_{4}$, 5-nucleotidase $\left(0.02\right.$ units $\left.\mathrm{mL}^{-1}\right)$ and bovine heart phosphodiesterase $\left(0.002\right.$ units $\left.\mathrm{mL}^{-1}\right)$, and appropriate concentration of the test compound in ethanol and Tris- $\mathrm{HCl}$ buffer ( $\left.\mathrm{pH} 7.5,50 \mathrm{mmol} \mathrm{L}^{-1}\right)$, in a final volume of $1 \mathrm{~mL}$. The reagents comprising $0.4 \mathrm{~mL}$ acid molybdate solution, $0.4 \mathrm{~mL}$ Elon reducing agent, and $1.0 \mathrm{~mL} \mathrm{H}_{2} \mathrm{O}$, to give a final volume of $3.0 \mathrm{~mL}$, were mixed in an incubation vessel, and the reaction was initiated by addition of cAMP and allowed to proceed for 20 min over a $30{ }^{\circ} \mathrm{C}$ water bath. The reaction was terminated by addition of $0.2 \mathrm{~mL} 5 \%$ trichloroacetic acid. The solution was then vortexed and centrifuged at $800 \times g$ for $10 \mathrm{~min}$. Color was allowed to develop for $20 \mathrm{~min}$ and absorbance was measured at $660 \mathrm{~nm}$. The results given in Table II are expressed as the concentration of inhibitor giving $50 \%$ inhibition $\left(I C_{50}\right)$ of the cAMP-dependent PDE activity. The $I C_{50}$ value was determined from plot of percentage inhibition $v s$. varying concentration of the inhibitor compounds $4 \mathbf{a}-\mathbf{j}$ and $5 \mathbf{a}-\mathbf{j}$. To validate the enzyme assay method, the $I C_{50}$ value of theophylline as a standard inhibitor was also determined. 
T. P. Selvam and P. V. Kumar: Synthesis and cAMP-dependent phosphodiesterase inhibition of novel thiazoloquinazoline derivatives, Acta Pharm. 61 (2011) 103-113.

\section{RESULTS AND DISCUSSION}

\section{Chemistry}

The series of heterocycles $\mathbf{4} \mathbf{a}-\mathbf{j}$ and $\mathbf{5 a}-\mathbf{j}$ were synthesized by the reaction of $\mathbf{3}$ with appropriate aromatic aldehyde and $p$-nitroaniline in the presence of anhydrous sodium acetate and DMF as it is presented in Scheme 1 . The IR, ${ }^{1} \mathrm{H}$ NMR, mass spectroscopy and elemental analyses for the new compounds were in accord with the assigned structures. The IR spectra of compounds $4 \mathbf{a}-\mathbf{j}$ showed stretching bands of the keto group at 1715-1740 $\mathrm{cm}^{-1}$. In $5 \mathbf{a}-\mathbf{j}$, stretching and bending $\mathrm{NH}$ bands of thiazoloquinazoline moiety appear at 3300-3400 and 1300-1350 cm-1, respectively. The recorded IR spectra of compounds 5a-j showed no keto group bands. This clearly envisages that the keto group of $4 \mathbf{a}-\mathbf{j}$ is converted into secondary NH. In $5 \mathbf{a}-\mathbf{j}$, the NH signal of 3-(4-nitrophenyl)aminothiazoloquinazoline moiety appear at $\delta 7.26(\mathrm{~s}), 7.23(\mathrm{~s}), 7.29(\mathrm{~s}), 7.69$ (s), $7.34(\mathrm{~s}), 7.16(\mathrm{~s})$,

Table II. PDE inhibition and relative data of synthesized compounds

\begin{tabular}{ccc}
\hline Compd. & $I C_{50}\left(\mathrm{mmol} \mathrm{L}^{-1}\right)$ & Relative activity \\
\hline $\mathbf{4 a}$ & $2.10 \pm 0.09$ & 1.22 \\
$\mathbf{4 b}$ & $2.40 \pm 0.02$ & 1.35 \\
$\mathbf{4} \mathbf{c}$ & $2.33 \pm 0.09$ & 1.34 \\
$\mathbf{4 d}$ & $2.28 \pm 0.02$ & 1.27 \\
$\mathbf{4} \mathbf{e}$ & $2.09 \pm 0.08$ & 1.22 \\
$\mathbf{4 f}$ & $2.11 \pm 0.08$ & 1.21 \\
$\mathbf{4 g}$ & $2.13 \pm 0.08$ & 1.28 \\
$\mathbf{4 h}$ & $2.32 \pm 0.08$ & 1.32 \\
$\mathbf{4} \mathbf{i}$ & $2.41 \pm 0.03$ & 1.35 \\
$\mathbf{4 j}$ & $2.64 \pm 0.02$ & 1.47 \\
$\mathbf{5 a}$ & $1.98 \pm 0.03$ & 1.09 \\
$\mathbf{5 b}$ & $2.12 \pm 0.04$ & 1.20 \\
$\mathbf{5} \mathbf{c}$ & $2.09 \pm 0.05$ & 1.18 \\
$\mathbf{5 d}$ & $2.03 \pm 0.04$ & 1.14 \\
$\mathbf{5 e}$ & $1.44 \pm 0.02$ & 0.87 \\
$\mathbf{5 f}$ & $1.34 \pm 0.09$ & 0.84 \\
$\mathbf{5 g}$ & $1.64 \pm 0.04$ & 0.93 \\
$\mathbf{5 h}$ & $2.07 \pm 0.06$ & 1.18 \\
$\mathbf{5 i}$ & $2.07 \pm 0.03$ & 1.17 \\
$\mathbf{5 j}$ & $1.52 \pm 0.05$ & 0.87 \\
Theophylline & $1.72 \pm 0.09$ & 1 \\
\hline & &
\end{tabular}

Solvent used: DMSO

$I C_{50}$ values were determined as described in the experimental section. Mean $\pm \mathrm{SD}, n=3$. 
7.79 (s), 7.67 (s), 7.42 (s) and 7.19 (s) ppm, respectively. The presence and position of NH signal in the ${ }^{1} \mathrm{H}$ NMR spectra of final compounds conform with the secondary NH proton in thiazoloquinazoline moiety. This clearly envisages that thiazole-3-one moiety was involved in 3-(4-nitrophenyl)amino formation. Mass spectra showed accurate molecular ion peaks at $m / z 300,478$ and 528 for $3,4 \mathrm{~h}$ and $5 \mathbf{e}$, resp. All these facts clearly demonstrated that the $3^{\text {rd }}$ position of keto group in thiazole ring was converted into secondary amino group, as indicated in Sheme 1 and conforming with the proposed structures $\mathbf{5 a - j}$.

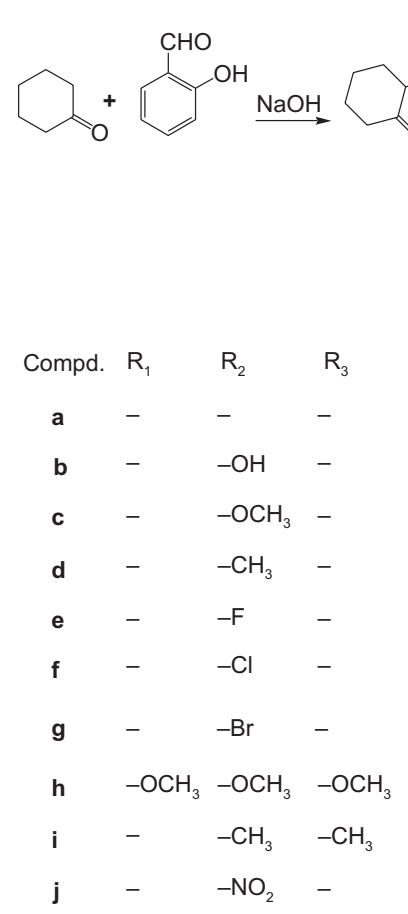

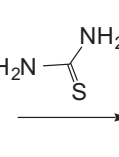<smiles>Oc1ccccc1C1NC(=S)NC2=C1CCCC2</smiles>

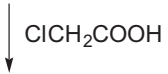<smiles>O=C1CSC2=NC3=CCCCC3=C(C3CCCCC3)N12</smiles><smiles>[R]c1cc(C=CC)cc([R])c1C</smiles><smiles>[R]c1cc(C2SC3=NC4=C(CCCC4)C(c4ccccc4O)N3C2O)cc([R])c1C</smiles><smiles>CC(CO)CN(C)C</smiles><smiles></smiles><smiles>NCCO[N+](=O)[O-]</smiles><smiles></smiles>

Scheme 1 


\section{PDE inhibition activity}

The target compounds were evaluated for their ability to inhibit bovine heart phosphodiesterase with theophylline chosen as a standard for comparison (Table II). All the compounds were found to exhibit PDE inhibitory activity. Despite the fact that this assay is less sensitive compared to radioisotope PDE assays and likely to underestimate the relative inhibitory potencies of the test compounds, the results of this evaluation indicate that the quinazoline derivatives are still more potent inhibitors of PDE than theophylline.

Compounds having thiazolo(2,3-b)quinazolin-3(2H)-one $(4 \mathbf{a}-\mathbf{j})$ and thiazoloquinazoline (5a-j) ring systems were all found to exhibit promising PDE inhibitory activity with $I C_{50}$ in the range 1.34-2.64 $( \pm 0.09) \mathrm{mmol} \mathrm{L}^{-1}$ and activity relative to theophylline of 0.84-1.47. Among them 6,7,8,9-tetrahydro-5H-5-(2'-hydroxyphenyl)-2-(4'-chlorobenzyline)-3-(4-nitrophenylamino)thiazoloquinazoline (5f) was found to be most potent, with relative activity of 0.8 compared to the standard, whereas for both compounds 6,7,8,9-tetrahydro-5H-5-(2'-hydroxyphenyl)-2-(4'-fluorobenzylidine)-3-(4-nitrophenylamino)thiazoloquinazoline (5e) and 6,7,8,9-tetrahydro-5H-5-(2'-hydroxyphenyl)-2-(4'-nitrobenzylidine)-3-(4-nitrophenylamino)thiazoloquinazoline (5j) relative reactivity was found to be 0.87 .

The electronic nature of the substituent groups at second positions in thiazole nucleus led to significant variation in PDE inhibitory activity. In both series the presence of an electron-withdrawing group (chloro, fluoro, nitro, bromo) in the thiazoloquinazoline nucleus resulted in higher activity. For example, 4-nitrophenyl amino substitution in thiazoloquinazoline nucleus (5a-j) increased activity; the order of activity was $\mathrm{Cl}>\mathrm{F}>\mathrm{NO}_{2}>$ $\mathrm{Br}>$ electron donating group. Compound $\mathbf{5 f}, \mathbf{5 e}, \mathbf{5 j}$ and $\mathbf{5 g}$ showed higher activity than the reference drug theophylline.

\section{CONCLUSION}

In summary, the present study has demonstrated that fused thiazoloquinazolines are novel inhibitors of phosphodiesterase and may potentially act as smooth muscle relaxatory agents. Further work is in progress to estabilish this observation in different animal models and find selective inhibitors of PDE 4, which may impact strategies for bronchodilator activity.

\section{REFERENCES}

1. W. J. Roesler, J. G. Graham, R. Kolen, D. J. Klemm and P. J. Mc Fie, The cAMP response element binding protein synergies with other transcription factors to mediate cAMP responsiveness, J. Biol. Chem. 270 (1995) 8225-8232; DOI: 10.1074/jbc270.14.8225.

2. G. S. McKnight, Cyclic AMP second messenger system, Curr. Opin. Cell Biol. 3 (1991) 213-217.

3. S. S. Taylor, S. A. Buechler and W. Yonemoto, cAMP-dependent protein kinase: framework for a diverse family of regulatory enzymes, Annu. Rev. Biochem. 59 (1990) 971-1005. 
T. P. Selvam and P. V. Kumar: Synthesis and cAMP-dependent phosphodiesterase inhibition of novel thiazoloquinazoline derivatives, Acta Pharm. 61 (2011) 103-113.

4. R. W. Butcher and E. W. Sutherland, Adenosine 3,5-phosphate in biological materials. I. Purification and properties of cyclic 3,5-nucleotide phosphodiesterase and use of this enzyme to characterize adenosine 3,5-phosphate in human urine, J. Biol. Chem. 237 (1962) 1244-1250.

5. A. Hatzelmann, E. J. Morcillo, G. Lungarella, S. Adnot, S. Sanjar, R. Beume, C. Schudt and H. Tenor, The preclinical pharmacology of roflumilaste - A selective, oral phosphodiesterase 4 inhibitor in development for chronic obstructive pulmonary disease, Pulm. Pharmacol. Ther. 23 (2010) 235-256; DOI: 10.1016/j.pupt.2010.03.011.

6. W. Jiang, J. Guan, M. J. Macielag, S. Zhang, Y. Qiu, P. Kraft, S. Bhattacharjee, T. M. John, D. H. Johnson, S. Lundeen and Z. Sui, Pyrroloquinolone PDE5 inhibitors with improved pharmaceutical profiles for clinical studies on erectile dysfunction, J. Med. Chem. 48 (2005) 2126-2133; DOI: 10.1021/jm0401098.

7. A. Daugan, P. Grondin, C. Ruault, A. C. Le Monnier de Gouville, H. Coste, J. Kirilovsky, F. Hyafil and R. Labaudinière, The discovery of tadalafil: A novel and highly selective PDE5 inhibitor. 1: 5,6,11,11a-Tetrahydro-1H-imidazo[1',5':1,6]pyrido[3,4-b]indole-1,3 (2H)-dione Analogues, J. Med. Chem. 46 (2003) 4525-4532; DOI: 10.1021/jm030056e.

8. A. Daugan, P. Grondin, C. Ruault, H. Coste, J. Kirilovsky, F. Hyafil and R. Labaudinière, The discovery of tadalafil: A novel and highly selective PDE5 inhibitor. 2: 2, 3, 6, 7, 12, 12a-hexahydropyrazino [1',2':1,6] pyrido[3,4-b] indole-1,4-dione analogues, J. Med. Chem. 46 (2003) 4533-4542; DOI: $10.1021 / j m 0300577$.

9. A. Martínez, A. Castro, C. Gil, M. Miralpeix, V. Segarra, T. Doménech, J. Beleta, J. M. Palacios, H. Ryder, X. Miró, C. Bonet, J. M. Casacuberta, F. Azorín, B. Piña and P. Puigdoménech, Benzyl derivatives of 2, 1, 3-benzo- and benzothieno [3,2-a] thiadiazine 2,2-dioxides: First phosphodiesterase 7 inhibitors, J. Med. Chem. 43 (2000) 683-689; DOI: 10.1021/jm990382n.

10. A. J. Duplantier, C. J. Andresen, J. B. Cheng, V. L. Cohan, C. Decker, F. M. DiCapua, K. G. Kraus, K. L. Johnson, J. W. Watson, R. T. Wester and A. S. Williams, 7-Oxo-4,5,6,7-tetrahydro-1H-pyrazolo[3,4-c] pyridines as novel inhibitors of human eosinophil phosphodiesterase, J. Med. Chem. 41 (1998) 2268-2277; DOI: 10.1021/jm9800090.

11. D. P. Rotella, Z. Sun, Y. Zhu, J. Krupinski, R. Pongrac, L. Seliger, D. Normandin and J. E. Macor, N-3-substituted imidazoquinazolinones: potent and selective PDE5 inhibitors as potential agents for treatment of erectile dysfunction, J. Med. Chem. 43 (2000) 1257-1263. DOI: 10.1021/jm000081+.

12. D. P. Rotella, Z. Sun, Y. Zhu, J. Krupinski, R. Pongrac, L. Seliger, D. Normandin and J. E. Macor, Optimization of substituted N-3-benzylimidazoquinazolinone sulfonamides as potent and selective PDE5 inhibitors, J. Med. Chem. 43 (2000) 5037-5043; DOI: 10.1021/jm000336j.

13. A. A. Bekhit, N. S. Habib and A. El-Din, Synthesis and antimicrobial evaluation of chalcone and syndrome derivatives of 4(3H)-quinazolinone, Boll. Chim. Farm. 140 (2001) 297-301.

14. B. Maggio, G. Daidone and D. Raffa, Synthesis and pharmacological study of ethyl 1-methyl-5-(substituted 3,4-dihydro-4-oxoquinazolin-3-yl)-1H-pyrazole-4-acetates, Eur. J. Med. Chem. 36 (2001) 737-742.

15. B. Lasztoczi, R. Kovacs and L. Nyikos, A glutamate receptor subtype antagonist inhibits seizures in rat hippocampal slices, Neuroreport 13 (2002) 351-356.

16. A. Lopez-Farre, J. A. Rodriguez-Feo and E. Garcia-Colis, Reduction of the soluble cyclic GMP vasorelaxing system in the vascular wall of stroke-prone spontaneously hypertensive rats effect of the alpha1-receptor blocker doxazosin, J. Hypertens. 20 (2002) 463-470.

17. R. Sharma, S. Kumar and H. K. Pujari, Reaction of 3,4,5,6,7,8-hexahydro-4-phenylquinazoline-2-thione with chloroacetic acid, Indian J. Chem. 30B (1991) 425-426. 
T. P. Selvam and P. V. Kumar: Synthesis and cAMP-dependent phosphodiesterase inhibition of novel thiazoloquinazoline derivatives, Acta Pharm. 61 (2011) 103-113.

$S A \check{Z} E T A K$

Sinteza i cAMP-ovisna inhibicija fosfodiesteraze novih derivata tiazolokinazolina

THEIVENDREN PANNEER SELVAM I PALANIRAJAN VIJAYARAJ KUMAR

U radu je opisana sinteza serije 6,7,8,9-tetrahidro-5H-5-(2'-hidroksifenil)-2-(4'-supstituiranih benzilidin)tiazolo(2,3-b)kinazolin-3(2H)-ona (4a-j) i 6,7,8,9-tetrahidro-5H-5-(2'-hidroksifenil)-2-(4'-supstituiranih benzilidin)-3-(4-nitrofenilamino)tiazolokinazolina (5a-j) prema objavljenoj metodi te ispitano njihovo inhibitorno djelovanje na fosfodiesterazu. Svi testirani spojevi pokazuju dobro djelovanje. Proučavan je i odnos strukture i djelovanja. U obje serije spojeva, elektron-odvlačeći supstituenti doprinose jačem djelovanju. Među ispitivanim spojevima pronađeno je da 6,7,8,9-tetrahidro-5H-5-(2'-hidroksifenil)-2-(4'-fluorobenzilidine)-3-(4-nitrofenilamino)tiazolokinazolin (5e), 6,7,8,9-tetrahidro-5H-5-(2'-hidroksifenil)-2-(4'-nitrobenzilidine)-3-(4-nitrofenilamino)tiazolokinazolin (5j) i 6,7,8,9-tetrahidro-5H-5-(2'-hidroksifenil)-2-(4'-klorobenzilidin)-3-(4-nitrofenilamino)tiazolokinazolin (5f) imaju jače djelovanje od teofilina ( $I C_{50} \mathrm{u} \mathrm{mmol} \mathrm{L} \mathrm{m}^{-1} 1,34 \pm 0,09$ za $5 \mathbf{f}$, $1,44 \pm 0,02$ za $5 \mathbf{e}, 1,52 \pm 0,05$ za $5 \mathbf{j}$ nasuprot $1,72 \pm 0,09$ za teofilin).

Ključne riječi: tiazolokinazolin, benzilidinetiazolokinazolin, nitrofenilaminotiazolokinazolin, inhibicija fosfodiesteraze, SAR

Department of Biotechnology Acharya Nagarjuna University, Guntur-522510, India

Department of Pharmacy, College Sadaya, International University, Jalan Menara Gading 56000 Cheras Kuala Lumpur, Malaysia 